Rev. Int. Contam. Ambie. 33 (1) 21-35, 2017

DOI: 10.20937/RICA.2017.33.01.02

\title{
COMPORTAMIENTO TEMPORAL Y ESPACIAL DEL BOSQUE RIBEREÑO EN EL CURSO BAJO DEL RÍO PORTOVIEJO Y LA QUEBRADA CHILÁN, PROVINCIA DE MANABÍ, ECUADOR
}

\author{
Argenis de Jesús MONTILLA PACHECO ${ }^{1 *}$ y Henry Antonio PACHECO GIL ${ }^{2}$
}

${ }^{1}$ Departamento Central de Investigación, Universidad Laica Eloy Alfaro de Manabí. Ciudadela Universitaria vía a San Mateo, Manta, Provincia de Manabí, República del Ecuador. C. P. 130802

${ }^{2}$ Instituto de Investigaciones, Universidad Técnica de Manabí. Avenida Urbina y Che Guevara, Portoviejo, Provincia de Manabí, República del Ecuador, C. P. 130105

*Autor para correspondencia: argenismontilla@hotmail.com

(Recibido julio 2015; aceptado marzo 2016)

Palabras clave: restauración ecológica, tecnologías SIG, agricultura y ganadería, reemplazo de bosques

\section{RESUMEN}

El objetivo de este trabajo fue estudiar y comparar, en términos espaciales y temporales, la cobertura de bosques ribereños en el curso bajo del río Portoviejo y la Quebrada Chilán mediante la aplicación de herramientas de información geográfica, sensores remotos y monitoreos de campo. Estos bosques se desarrollan a lo largo de dos cursos de agua asentados en cuencas contiguas, que desembocan en el Pacífico ecuatoriano, entre Jaramijó y San Clemente. Los resultados muestran que en el curso bajo del río Portoviejo los bosques ribereños tuvieron bajos porcentajes de cobertura en los años 1998, 2002, 2009, 2011 y 2013, mientras que en la quebrada Chilán aumentaron significativamente desde 1998 hasta 2014, cubriendo ambos márgenes del cauce en todo su recorrido. El creciente desarrollo agrícola y ganadero es causa principal por la cual desapareció gran parte del bosque ribereño en los márgenes del río Portoviejo. En la quebrada Chilán, en contraste, no se aprecia actividad económica que conlleve a la ocupación espacial y al reemplazo del bosque. Sobre la base de los resultados encontrados y por la importancia ecológica de estas áreas naturales, se discuten y plantean estrategias para la conservación de bosques ribereños en la quebrada Chilán, como también para restaurar áreas de bosques ribereños en el curso del río Portoviejo reemplazadas por cultivos y pastizales. Dentro de las propuestas de restauración se incluyen acciones tendientes a recuperar su trayectoria original, tomando como referencia pequeños parches de vegetación del mismo tipo que aún se conservan en el área de estudio.

Key words: ecological restoration, GIS technologies, agriculture and livestock, forest replacement

\begin{abstract}
The aim of this work was to study and compare, in spatial and temporal terms, the coverage of riparian forests in the lower reaches of the Portoviejo River and the ravine Chilán through the application of tools of geographical information systems, remote sensing and field monitoring. These forests are developed along two streams seated in adjacent basins, which flow into the Ecuadorian Pacific between Jaramijó
\end{abstract}


and San Clemente. The results show that in the lower reach of the Portoviejo River, riparian forests had low rates of coverage in the years 1998, 2002, 2009, 2011 and 2013, while in the Chilán ravine, the rates of coverage increased significantly from 1998 to 2014, covering both riverbanks all the way. The growing agricultural and livestock developments are the main reasons why much of the riverine forest on the banks of Portoviejo River has disappeared. In contrast, in the Chilán ravine, there is no ongoing economic activity that may lead to spatial occupation and replacement of the forest. Based on the results found and the ecological importance of these natural areas, strategies for the conservation of riparian forests in the Chilán ravine are discussed and proposed, as well as strategies to restore areas of riparian forests throughout the Portoviejo River that have been replaced by crops and pastures. Among the proposals for restorative efforts, actions aimed at recovering its original trajectory are included, taking as reference small patches of vegetation of the same type that still remain in the study area.

\section{INTRODUCCIÓN}

Los bosques de galería o ribereños se presentan como comunidades exuberantes en relación con su entorno. Se desarrollan en los márgenes de los cauces de ríos, arroyos y canales, formando una estrecha franja que funciona en muchas ocasiones como corredores de fauna al comunicar comunidades vegetales aisladas (Treviño et al. 2001). Los bosques de ribera constituyen una comunidad muy variada desde el punto de vista de su fisionomía y estructura, de modo que esta heterogeneidad estructural y funcional, sumada a la poca superficie ocupada, y a la importancia ecológica que representan, son elementos clave para la conservación de la diversidad biológica (Ruiz 2004).

Sobre la situación de los bosques en el Ecuador, la Secretaría Nacional de Planificación y Desarrollo (SENPLADES 2013), señala que aún con los esfuerzos realizados por alcanzar la protección de este patrimonio natural, la deforestación ocasionada por la expansión de la frontera agrícola continúa siendo una de las principales preocupaciones del país. Ante tales afirmaciones, es interesante indagar acerca de la situación en particular del bosque de ribera, que es uno de los muchos tipos que conforman el conjunto de bosques en Ecuador.

Actualmente, gracias a los avances en percepción remota es posible desarrollar estudios relacionados con la vegetación ribereña, pues son muy apropiados para analizar la vulnerabilidad de la cobertura vegetal en los ecosistemas terrestres, pudiendo detectar problemas específicos para proyectar eventos futuros con la posibilidad de implementar estrategias de mitigación y con el potencial para desarrollar alertas tempranas (Jacques et al. 2014).
De igual forma, señalan Sun et al. (2014) que hoy se dispone de tecnologías de la información geográfica para estudiar la cobertura vegetal, la producción de biomasa y para diversas aplicaciones en la agricultura de precisión. Estos instrumentos facilitan al agricultor información precisa sobre las variaciones agroecológicas de las parcela para que el territorio se gestione optimamente y conseguir así la máxima producción posible en toda su extensión. Dichos instrumentos se han puesto en práctica con buenos resultados en países como China, Ghana, Malasia, Estados Unidos de América y Brasil, entre otros.

Lo antes mencionado es particularmente importante en este momento, cuando el crecimiento demográfico humano es muy significativo en el área de estudio (Fig. 1), pues este crecimiento y las actividades de uso actual del suelo crean fuertes presiones sobre la vegetación (Treviño 2001), a través de la extracción forestal o por la producción agrícola, siendo esta última la actividad de mayor

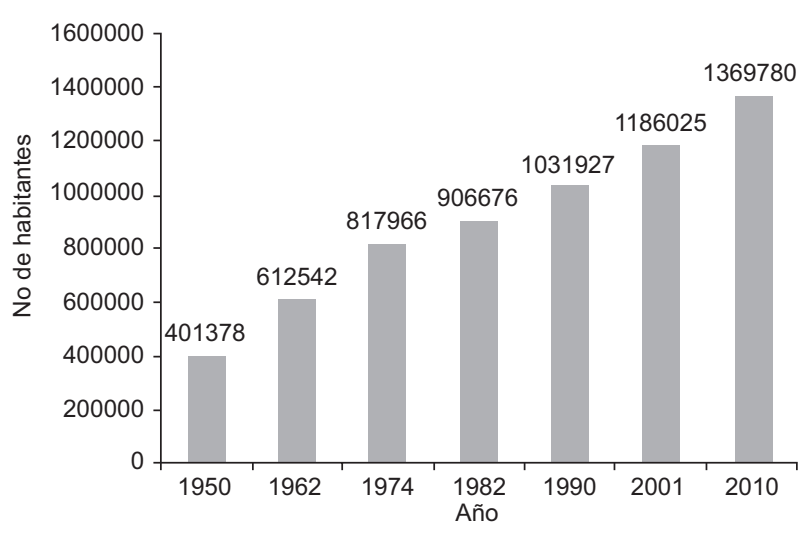

Fig. 1. Crecimiento de la población en la provincia de Manabí, según los datos de los censos generales de población y vivienda. Fuente: INEC (2010). 
relevancia en la cuenca del río Portoviejo (Thielen et al. 2015).

Finalmente, se espera que los aspectos metodológicos empleados y los resultados de este trabajo puedan ser útiles para el desarrollo de investigaciones en otras regiones de Ecuador y Suramérica, donde las metas relacionadas con la conservación de los ecosistemas naturales no han cumplido con los Objetivos del Milenio de las Naciones Unidas. Para ello, se planteó la agenda Post 2015 con los Objetivos de Desarrollo Sostenible, en la que se contempla la medición de indicadores directos sobre la deforestación de bosques. Por lo que en esta investigación se pretende analizar el comportamiento espacial y temporal de los bosques de ribera, generar información de interés aplicable en futuros programas de restauración, así como sugerir acciones y estrategias para su recuperación y para frenar los procesos de degradación de los mismos.

\section{CARACTERÍSTICAS DEL ÁREA DE ESTUDIO}

El área de estudio se localiza en las cuencas bajas del río Portoviejo y de la quebrada Chilán, ubicadas en la parte noroccidental de la República del Ecuador y forman parte de los espacios contiguos a la costa del Pacífico, siendo jurisdicción de la provincia de Manabí. El área está comprendida entre las coordenadas $0^{\circ} 47^{\prime} 16^{\prime \prime}$ y $1^{\circ} 0$ ' $44^{\prime \prime}$ de latitud sur y $80^{\circ} 25^{\prime}$ $41^{\prime \prime}$ y $80^{\circ} 35^{\prime}$ 06" de longitud oeste (Fig. 2).
Las principales actividades humanas, concentradas en la cuenca baja del río Portoviejo son la agricultura y la ganadería, la primera, expresada en la práctica de diversos cultivos como maíz, plátano y arroz, y algunos cultivos hortícolas. La ganadería se orienta a la producción de carne y leche a partir del aprovechamiento de pastos cultivados en lugares que anteriormente estaban ocupados por ecosistemas boscosos.

Las características del relieve están definidas por una llanura de suaves pendientes donde impera un clima megatérmico semiárido (Gobierno Autónomo Descentralizado Municipal del Cantón Montecristi 2011), con valores de precipitación anual cercanos a los $300 \mathrm{~mm}$, razón por la cual se caracteriza como un ambiente semidesértico (Cedeño 2010). Los bajos niveles de precipitación imponen la presencia, en su mayoría, de una vegetación dominada por el bosque seco tropical. En general, se producen dos estaciones bien diferenciadas, una húmeda entre enero y abril y una seca entre mayo y diciembre (SENPLADES 2010a).

La temperatura media anual está alrededor de $26^{\circ} \mathrm{C}$ (Pourrut 1983), pero desciende durante el período seco debido a la influencia que ejercen las masas de aire frío que acompañan a la corriente de Humboldt (Milton 2012). Otra corriente, llamada Tropical, viene del norte y oeste del Pacífico y se asocia a eventos ENOS (El Niño Oscilación Sur) responsables de importantes precipitaciones en forma cíclica (Cedeño 2010, Thielen et al. 2015).

La hidrografía está conformada por una red de drenaje de distinto régimen. Así, la quebrada Chilán es un curso de agua intermitente, pues sólo presenta

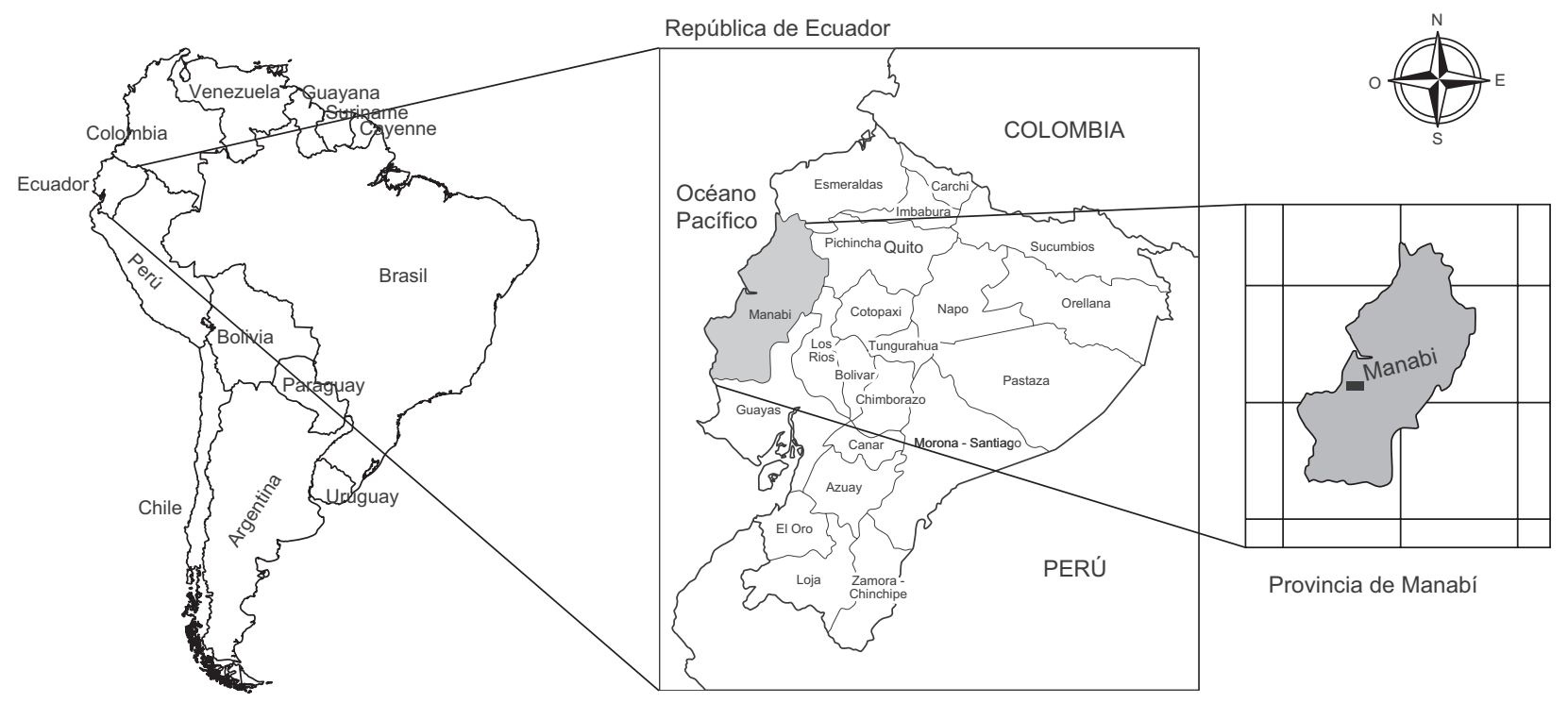

Fig. 2. Localización del área de estudio. 
caudal unas pocas horas después de producirse la lluvia, fenómeno característico de ambientes semiáridos. Por su parte, el río Portoviejo es de régimen permanente, y aunque tiene variaciones de caudal a lo largo del año, nunca llega a secarse, pues es alimentado por las precipitaciones que ocurren en la cuenca alta, donde los montos se aproximan a los $2000 \mathrm{~mm}$ anuales, en un período de lluvia mucho más prolongado que en la cuenca baja. $\mathrm{Al}$ respecto, el régimen hidrológico de los cursos de agua en las cuencas bajas estudiadas corresponde al régimen pluvial tropical árido de la costa meridional, que se caracteriza por una pluviometría inferior a $500 \mathrm{~mm}$ anuales, y el flujo fluvial es marcadamente temporal (Pourrut 1995).

Respecto a la fauna, debe señalarse que Ecuador es un país con una gran biodiversidad, no solamente de flora, sino también de fauna. Cada región, dadas sus condiciones ecológicas es el hábitat de numerosas especies. Es así como en el área de estudio se ha reportado la presencia de distintas especies de aves y reptiles. Por ejemplo, en zonas pantanosas cercanas a la costa habitan caimanes, y en ambientes boscosos viven el oso hormiguero (Myrmecophaga tridactyla), el jaguar (Panthera onca), el perezoso (Choloepus didactylus), el loro orejiamillo (Ognorhynchu icterotis), la gallineta (Gallinula chloropus), la pava de monte (Penelope jacquacu) y el guatusa (Dasyprocta fuliginos), entre otros.

\section{FUNDAMENTACIÓN TEÓRICA Y LEGAL DE LA INVESTIGACIÓN}

En la República del Ecuador, las investigaciones que puedan desarrollarse en torno al conocimiento del ambiente, la ecología y los recursos naturales deben responder al sistema de planificación propuesto por el Estado a través de diferentes agendas y planes de desarrollo (SENPLADES 2010b). En ese contexto, la presente investigación se fundamenta en los planteamientos establecidos en la Constitución Nacional de la República del Ecuador (2008), específicamente en el artículo 280, que textualmente señala que "EI Plan Nacional de Desarrollo es el instrumento al que se sujetarán las políticas, programas y proyectos públicos, la programación y ejecución del presupuesto del Estado, la inversión y la asignación de los recursos públicos y la coordinación de las competencias exclusivas entre el Estado central y los gobiernos autónomos descentralizados. Su observancia será de carácter obligatorio para el sector público e indicativo para los demás sectores".
Igualmente este trabajo se enmarca dentro de lo que señala el Plan Nacional del Buen Vivir 2013-2017 elaborado por SENPLADES (2013), que persigue mejorar la calidad de vida de la población, fortalecer las capacidades y potencialidades de la ciudadanía y garantizar los derechos de la naturaleza, así como promover la sostenibilidad territorial y global, a partir del principio de armonía hombre-naturaleza. Este principio se basa en el reconocimiento de los derechos de la naturaleza y la garantía de un ambiente sano y sostenible, donde las actividades desarrolladas por los grupos humanos se hagan dentro de los límites biofisicos de los ecosistemas, asumiendo responsabilidad con las futuras generaciones. Además se corresponde con lo que persigue el objetivo 7 del Plan Nacional del Buen Vivir 2013-2017, específicamente el objetivo 7.2 literal m, donde se determina que el Estado debe fomentar la investigación y los estudios prospectivos sobre el uso sustentable y la conservación de la biodiversidad terrestre, acuática y marino-costera.

Otro plan que guarda relación con lo ambiental es el que lleva adelante la Corporación Forestal y Ambiental de Manabí (CORFAM), que procura mejorar las condiciones ambientales de la provincia con actividades de reforestación en las áreas más degradadas, $\mathrm{y}$ tiene como meta cubrir cerca de unas 500000 ha en los próximos años. Esta acción introduce sustanciales mejoras en el ambiente, que junto a las labores de concienciación, apunta hacia la búsqueda del equilibrio entre el desarrollo económico y la sustentabilidad ecológica.

\section{MÉTODOS}

El desarrollo de esta investigación implicó la aplicación de distintas metodologías tanto para los aspectos ecológicos y ambientales como para los aspectos socioeconómicos. En ese sentido, se utilizaron imágenes de satélite de Google Earth, SPOT y RapidEye que fueron procesadas utilizando tecnologías de la información geográfica para la obtención de información sobre los dos cursos de agua.

Las imágenes de Google Earth se interpretaron según la metodología propuesta por la Universidad Nacional de San Luis (2011), la cual se hace directamente y de manera visual utilizando los principios de la fotointerpretación. Se consideraron elementos de tonalidad, textura fotográfica y estructura, asociando lo observado en las imágenes, con elementos de la realidad por semejanza o analogía. Se emplearon elementos conocidos de una manera bastante precisa, ya que es posible tener una visión $3 \mathrm{D}$ del terreno, 
con muy buena resolución para el área de estudio en este caso. Para determinar la distribución espacial de los bosques de galería en la parte baja de la cuenca de la quebrada Chilán y del río Portoviejo se usaron de manera comparativa imágenes de los años 1998, 2002 y 2011.

Para el caso del satélite SPOT se procesaron imágenes de noviembre de 2009, a una resolución de $10 \mathrm{~m}$, que fueron corregidas geométrica y radiométricamente.

Las correcciones y procedimientos metodológicos empleados en general se detallan a continuación.

\section{Correcciones geométricas}

Las correcciones geométricas en las imágenes se realizaron en atención a lo que sugieren Chuvieco (2000) y Ambrosio et al. (2007), con el propósito de orientar la posición de los pixeles respecto al sistema de referencia cartográfica de Ecuador mediante el siguiente procedimiento:

a. La localización de puntos de control (GCP, por sus siglas en inglés). Para relacionar los valores de coordenadas al sistema cartográfico de referencia señalado se trabajó con las ortofotos del Instituto Geográfico Militar de Ecuador. Sobre el ortofotomapa se ubicaron 16 puntos claramente visibles en la imagen, distribuidos de manera tal que abarcasen equitativamente toda la cobertura. Estos puntos se corresponden con la intersección de vialidades, infraestructura y cursos de agua, garantizando así una identificación inequívoca tanto en el ortofotomapa como en la imagen.

b. Obtención de los parámetros de transformación de las coordenadas. Las coordenadas de la imagen fueron transformadas en coordenadas cartográficas. Para ello se usaron las ecuaciones polinómicas de segundo orden, generando una matriz de coeficientes calculada a partir de los puntos de control, usando el método de regresión por mínimos cuadrados. El objetivo en el cálculo de los coeficientes de la matriz es derivar las ecuaciones polinómicas para las cuales existe la menor cantidad de error cuando se usan para transformar las coordenadas de referencia de los puntos de control en coordenadas fuente.

Para verificar la precisión de la corrección se consideró el error medio cuadrático (EMC) como la distancia entre la posición de entrada (fuente) de un punto de control y la posición retransformada del mismo GCP. En otras palabras, es la diferencia entre la coordenada de salida deseada y la coordenada de salida real para el mismo punto, cuando el punto es transformado geométricamente. El error EMC se expresa como distancia en el sistema de coordenadas fuente. Si las coordenadas del archivo de los datos son las coordenadas fuente, entonces el error EMC es una distancia en unidades de pixel. Para este trabajo, siempre se obtuvo un EMC por debajo de 1, lo que significa que el pixel de referencia está por debajo de un pixel desplazado del pixel retransformado. Para este caso se determinó un pixel de $10 \mathrm{~m}$ de resolución, y por lo tanto el error siempre está por debajo de esta magnitud.

c. Remuestreo o reubicación de los pixeles. El último paso fue crear el archivo de salida. Dado que la malla (cuadrícula) de pixeles en la imagen fuente rara vez coincide con la malla de la imagen de referencia, los pixeles son remuestreados. Con este procedimiento se puede calcular los nuevos niveles digitales para el archivo de salida. Se remuestreó por el método de convolución cúbica, que usa los niveles digitales de 16 pixeles en una ventana de $4 \times 4$ para calcular el valor de salida con una función cúbica.

\section{Correcciones radiométricas}

Para aproximar la respuesta recibida por el sensor con el objeto real observado en la superficie terrestre se aplicaron métodos que tienden a eliminar la dispersión por sustracción. El método usado en esta ocasión fue el conocido como el del histograma mínimo. De acuerdo con Hum et al. (2014), este método se limita a substraer en cada banda el valor mínimo observado, ya que se supone que en una escena siempre pueden existir algunos pixeles en sombra total, que en ausencia de atmósfera no recibirían ni deberían reflejar ninguna energía de procedencia solar. Sin embargo, a objeto de hacer mejoras se consideró la metodología empleada por Song et al. (2001) y por Brizuela et al. (2007), para obtener el valor de dispersión para el infrarrojo medio y calcular el valor teórico de dispersión para el resto de las longitudes de onda.

Esta corrección radiométrica fue necesaria para este estudio, por cuanto los datos de la imagen fueron utilizados en el análisis de la cobertura de bosques, en la clasificación supervisada y en el cálculo del Índice Normalizado de Diferencia de Vegetación. Con este propósito fue necesario obtener la proporción de la energía reflejada que alcanza el sensor, conocida como radianza. Para ello se analizaron los valores del pixel de la imagen original como niveles digitales asociados a los registros eléctricos que percibió el sensor en el momento de la toma de la escena. Para transformar los valores de este código binario a 
valores en unidades de energía, fue necesario conocer los parámetros de compensación del sensor.

El grupo de datos SPOT-5 nivel 1-A contiene niveles digitales (ND) calibrados que son cuantificados como números enteros de 8 bits sin signo. Así se entiende en Gond et al. (2004) al plantear que los ND calibrados se convierten a radianza (la proporción de energía que recibe el sensor) usando la pendiente, los parámetros de compensación a partir del coeficiente de conversión de unidad, disponibles para cada banda en los metadatos, así como los parámetros de ganancia conocidos.

\section{Ortocorrección}

La imagen SPOT fue ortorrectificada siguiendo los criterios de Compton et al. (2004) usando los parámetros orbitales y un modelo digital del terreno a $10 \mathrm{~m}$ de resolución, construido por interpolación de las curvas de nivel del Instituto Geográfico Militar.

\section{Imagen RapidEye}

Se obtuvo la imagen RapidEye de noviembre de 2009 , con una resolución espacial de $5 \mathrm{~m}$ y un nivel de procesamiento $3 \mathrm{~A}$, que incluye correcciones radiométricas y geométricas del producto estándar, que ya viene ortorrectificado, con un Modelo Digital de Elevación (DEM por sus siglas en inglés) y Radar de Misión Topográfica (SRTM, por sus siglas en inglés), listo para ser incorporado en un Sistema de Información Geográfica (SIG). Sobre las imágenes SPOT y RapidEye se hizo interpretación visual en color verdadero y falso color, además del tratamiento digital para el cálculo del Índice Normalizado de Diferencias de Vegetación (NDVI, por sus siglas en inglés) y determinar la cobertura del bosque ribereño en los sectores estudiados.

\section{Cálculo del Índice Normalizado de Diferencias de Vegetación}

El Índice Normalizado de Diferencias de Vegetación (INDV) se usó, de acuerdo con los planteamientos de Baihua y Burgher (2015), para determinar los cambios a largo plazo en las características de la vegetación, recomendado por los autores para zonas semiáridas en condiciones ecológicas similares a las presentes en la zona estudiada. El INDV se obtiene con la expresión:

$\mathrm{INDV}=(\mathrm{PIR}-\mathrm{VIS}) /(\mathrm{PIR}+\mathrm{VIS})$,

Siendo:

INDV = Índice Normalizado de Diferencias de Vegetación
PIR $=$ Banda infrarrojo cercano

$\mathrm{VIS}=$ Banda roja

Con la aplicación de esta ecuación se generó una imagen con valores normalizados entre 1 y -1 , donde los valores negativos indican zonas desprovistas de vegetación, y los tendientes a 1 áreas con cobertura vegetal espesa.

\section{Aspectos socioeconómicos}

Otros aspectos, específicamente los de tipo socioeconómico, se abordaron sobre la base de información recolectada en campo y de fuentes bibliográficas. Además, se llevaron a cabo entrevistas no estructuradas a informantes clave (residentes en distintos sectores del área de estudio), con las que se indagó sobre las actividades económicas de cada sector y las prácticas agrícolas empleadas, entre otras cuestiones.

\section{Registros de eventos ocurridos}

Los registros de eventos con riesgos de desastres socionaturales ocurridos se obtuvieron de la base de datos Desinventar, desarrollada por La Red de Estudios Sociales en Prevención de Desastres en América Latina (La Red), en lenguaje MS Access con el objeto de ingresar de una manera estandarizada, consultar y procesar información sobre los desastres socionaturales y sus efectos. La Desinventar reporta eventos de desastres sobre unidades geográficas, pudiendo inventariarse los ocurridos desde 1970 a nivel de la mínima división política territorial, es decir la parroquia, Esta base se puede consultar gratuitamente y ya está actualizada hasta el 2015. Para alimentar la base de datos la información se obtuvo principalmente a partir de fuentes hemerográficas, de las Juntas Provinciales de Defensa Civil para el fenómeno El Niño 97-98, así como del Instituto Geofísico y Petroecuador, y de otras fuentes menores.

\section{Acciones y estrategias para recuperar el bosque de ribera y frenar los procesos que conducen a su degradación}

Para sugerir acciones y estrategias de recuperación del bosque ribereño, así como para coadyuvar a frenar los procesos de degradación y destrucción de los mismos, se partió de la información obtenida en observaciones de campo, pero también del análisis visual de las imágenes de satélite empleadas. En ese sentido, se recomendaron por sus condiciones actuales, algunos sectores, especialmente los más degradados y en los que la vulnerabilidad es mayor 
respecto a la ocurrencia de desbordamientos del cauce e inundaciones con potenciales afecciones a centros de población.

Para recomendar los sectores prioritarios a ser restaurados se tomó en cuenta el acceso a los lugares en los que hay remanentes de bosques con condiciones similares a los que han sido degradados, pues en ellos es posible encontrar plántulas de especies propias de la zona que son las más recomendables de emplear en actividades de restauración.

\section{RESULTADOS Y DISCUSIÓN}

\section{Comportamiento temporal y espacial del bosque ribereño en la quebrada Chilán}

El comportamiento temporal de los bosques ribereños muestra diferencias significativas de cobertura entre los años 2004 y 2013. En el primer año mencionado se observa la presencia abundante de colores claros en la imagen de Google Earth, lo que sugiere la ausencia de bosque y la dominancia de material sedimentario en el lecho fluvial. Por el contrario, se aprecia una buena cobertura del bosque ribereño en 2013, cuando prácticamente se puede inferir la red de drenaje por el verdor de la vegetación (Fig. 3).

Estos resultados son un claro indicador de la afectación al ecosistema bosque, por lo tanto se justifica la necesidad de desarrollar medidas de restauración y protección del mismo. Estas medidas son de especial importancia para la cuenca del río Portoviejo. Esta cuenca es vital para el suministro de servicios ecosistémicos como lo es el recurso hídrico para consumo humano y para las actividades agropecuarias de multipropósitos. Este recurso es dependiente de la presa Poza Honda que es alimentada por la cuenca del mencionado río.

Es posible que la abundancia de sedimentos en el cauce en 2004 esté determinada por la ocurrencia de crecidas torrenciales producto de las intensas precipitaciones registradas en el área de estudio en años anteriores. Tales precipitaciones estuvieron relacionadas con la manifestación del fenómeno El Niño, con eventos de gran magnitud en pocos años: el primero el de 1982-1983 y el segundo en 1997-1998, según reporta Zevallos (2004). Para el área de estudio se evidencia en los montos de precipitación de las estaciones Manta, Rocafuerte y Portoviejo (Fig. 4), donde además de los años indicados hubo también un incremento considerable de la precipitación en el período 2001-2002 con respecto a la precipitación media de largo plazo.

Los planteamientos anteriores se apoyan en los datos de los cuadros I y II pues existe una correlación directa de los grandes eventos lluviosos con sus respectivas consecuencias en la población humana. En ese sentido el número de muertos registrados durante el periodo de estudio según la base de datos Desinventar de la Red, fue de seis en la ciudad de Portoviejo, dos en la ciudad de Manta y dos en la

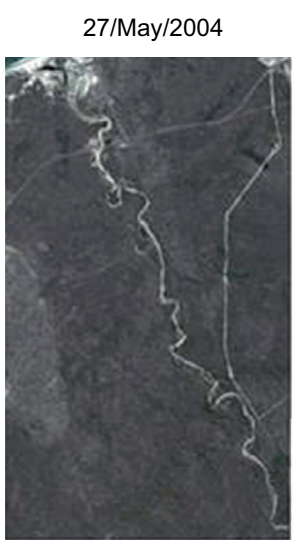

a

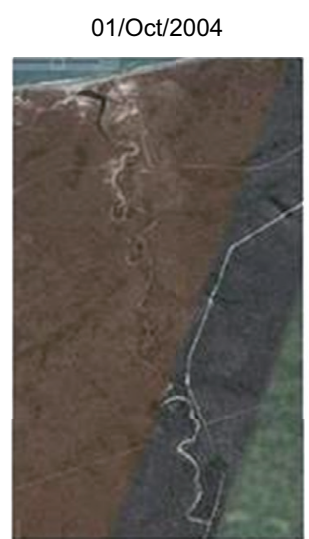

b

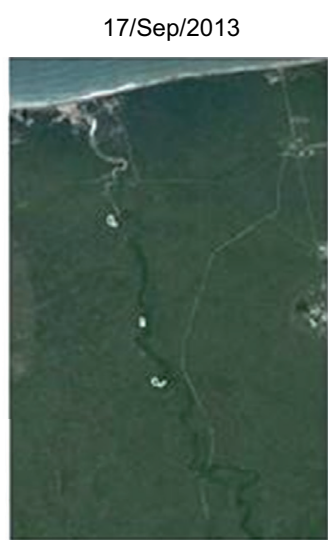

C

Fig. 3. Diferencia temporal de cobertura de bosques ribereños en la cuenca baja de la quebrada Chilán, Manabí, Ecuador. (a) El cauce del río está completamente despejado y definido en 2004, (b) El cauce del río se observa parcialmente, como consecuencia de la regeneración de la vegetación ribereña en 2009 y (c) El cauce del río no se puede apreciar debido a la regeneración de la cobertura del bosque ribereño en 2013. Fuente: Imágenes tomadas de Google Earth. 


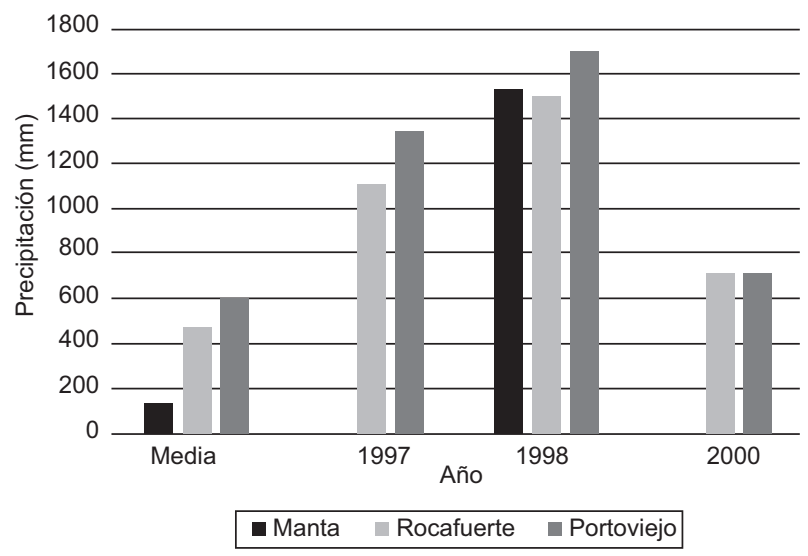

Fig. 4. Lluvia media y lluvia anual ( $\mathrm{mm}$ ) para las estaciones de Manta, Rocafuerte y Portoviejo. Obsérvese que en los años 1997, 1998 y 2002, los valores están muy por encima de la media, lo cual pone en evidencia la influencia del fenómeno El Niño en el incremento de las lluvias. Fuente: Instituto Nacional de Meteorología, Anuarios Meteorológicos 1991-2001. Quito, Ecuador.

ciudad de Montecristi, todos debido a deslizamientos de tierra. Así como catorce, cero y seis muertes respectivamente para las mismas ciudades, causadas por inundaciones, a consecuencia de las fuertes crecidas en épocas de la manifestación del fenómeno El Niño.

La anterior afirmación puede sustentarse también en Bolívar (2012), quien apunta que el Estero Chilán permanece seco casi todo año. Sin embargo, en invierno, cuando las lluvias son torrenciales, especialmente si hay un evento de El Niño, se presenta el transporte de enormes flujos de agua con importantes procesos de erosión. Estos flujos arrastran consigo grandes cantidades de sedimentos en suspensión y carga de fondo, afectando muchas veces viviendas, carreteras, puentes y otras obras de infraestructura. Por supuesto, la vegetación ribereña no escapa a esta afectación.

En el año 2013 la cobertura vegetal que bordea el cauce se había recuperado progresivamente hasta mostrar una estructura densa y compacta según se aprecia en la imagen de ese año. Tal recuperación guarda relación con la casi nula disponibilidad de agua para usos agrícolas, lo que limita o restringe los aprovechamientos agronómicos. De este modo, tras la presencia de lluvias extraordinarias queda abierta la posibilidad de que se produzcan procesos de regeneración natural de la vegetación ribereña, después de haber desaparecido por las grandes crecidas y desbordamientos de la quebrada. Esto pasó fundamentalmente en aquellos años en los que se manifiesta con mayor fuerza el fenómeno El Niño.

\section{Comportamiento temporal y espacial del bosque ribereño en el río Portoviejo}

Las imágenes utilizadas para identificar el comportamiento del bosque ribereño en el curso bajo del río (Fig. 5) muestran que en 1970 esta parte del curso ya estaba intervenida por actividades de ganadería y agricultura en ambas márgenes del cauce. Estas actividades se intensificaron a través del tiempo, tal como se aprecia en las imágenes posteriores, correspondientes a los años 2006, 2009 y 2014. Se cuantificó el cambio de uso del suelo de acuerdo con los tipos de cobertura señalados en el cuadro III establecidos por el MAE (2010). Se encontró que en el área de estudio desapareció un total de 5787.11 ha. de bosque entre los años

CUADRO I. AFECCIONES POR DESLIZAMIENTOS ENTRE LOS AÑOS 1997-1998 Y 20012002. EVENTOS REGISTRADOS EN LA BASE DE DATOS DESINVENTAR. LA RED DE ESTUDIOS SOCIALES EN PREVENCIÓN DE DESASTRES EN AMÉRICA LATINA (LA RED).

\begin{tabular}{llcccc}
\hline Años & Lugar & $N^{0}$ eventos & Muertos & Damnificados & Viviendas afectadas \\
\hline 1997 & & 1 & 2 & 30 & 0 \\
1998 & Portoviejo & 6 & 2 & 0 & 45 \\
2001 & & 2 & 0 & 0 \\
2002 & & 0 & 0 & 0 \\
\hline 1997 & & 0 & 0 & 0 & 0 \\
1998 & Montecristi & 3 & 3 & 64 & 4 \\
2001 & & 0 & 0 & 0 & 0 \\
2002 & & 0 & 0 & 0 & 0 \\
\hline 1997 & & 0 & 0 & 0 & 0 \\
1998 & Manta & 0 & 0 & 0 & 0 \\
2001 & & 0 & 0 & 0 & 190 \\
2002 & & 1 & 2 & 0 & 0 \\
\hline
\end{tabular}


CUADRO II. AFECCIONES POR INUNDACIONES ENTRE LOS AÑOS 1997-1998 Y 2001-2002. EVENTOS REGISTRADOS EN LA BASE DE DATOS DESINVENTAR. LA RED DE ESTUDIOS SOCIALES EN PREVENCIÓN DE DESASTRES EN AMÉRICA LATINA (LA RED).

\begin{tabular}{llcccc}
\hline Años & Lugar & N $^{\text {e eventos }}$ & Muertos & Damnificados & Viviendas afectadas \\
\hline 1997 & Portoviejo & 2 & 0 & 0 & 0 \\
1998 & & 4 & 11 & 528 & 148 \\
2001 & & 0 & 0 & 0 & 0 \\
2002 & & 3 & 3 & 1500 & 3280 \\
\hline 1997 & Montecristi & 2 & 0 & 5 & 0 \\
1998 & & 1 & 0 & 0 & 47 \\
2001 & & 0 & 0 & 0 & 0 \\
2002 & & 0 & 0 & 0 & 0 \\
\hline 1997 & Manta & 3 & 4 & 200 & 120 \\
1998 & & 3 & 2 & 0 & 0 \\
2001 & & 0 & 0 & 0 & 0 \\
2002 & & 2 & 0 & 0 & 113 \\
\hline
\end{tabular}

de 1990 y 2008, de las cuales 5541.24 (95.7\%), correspondieron a la cuenca del río Portoviejo. En la parte baja de esta cuenca el bosque ribereño fue completamente sustituido por diferentes tipos de cultivo, según se muestra en el cuadro III. El cambio máximo absoluto ocurrió en los años de 1990 al 2000, con una superficie total afectada de 2303.65 ha. En líneas generales, entre los años de 2000 y 2008 se presentó la máxima intensidad de deforestación con un total de 3110.23 ha, lo que se corresponde con una tasa anual de deforestación de $388.77 \mathrm{ha} /$ año equivalente a una pérdida de cobertura de bosques del $0.18 \%$ por año. Los cambios de uso del suelo más significativos fueron de bosque a mosaico agropecuario $(50.93 \%$,), seguido de pastizales $(27.03 \%)$ y cultivos anuales con 19.23 $\%$. Para este caso los mosaicos agropecuarios se diferencian de las otras coberturas por presentar una mezcla muy heterogénea de cultivos.

La combinación en falso color $(5,3,2)$ sobre la imagen RapidEye del año 2009 (Fig. 6), muestra un mosaico agropecuario en las proximidades del poblado de Rocafuerte, reflejando la presión antropogénica sobre las márgenes del río, donde ha desaparecido completamente el bosque ribereño. De igual forma, el INDV, correspondiente a la misma imagen (Fig. 7), refleja la ausencia de estructuras boscosas en las márgenes del río, lo cual es una evidencia contundente de la desaparición del bosque ribereño en el curso bajo del río Portoviejo.
01/Ene/1970

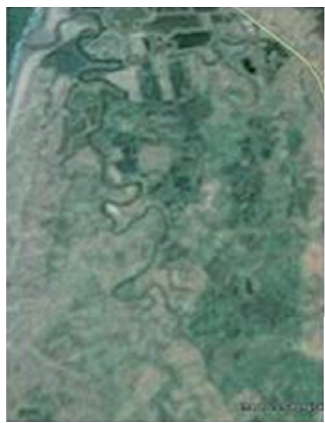

a
04/Ago/2006

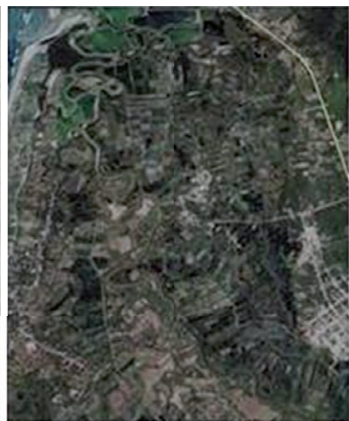

b
$11 / \operatorname{Sep} / 2014$

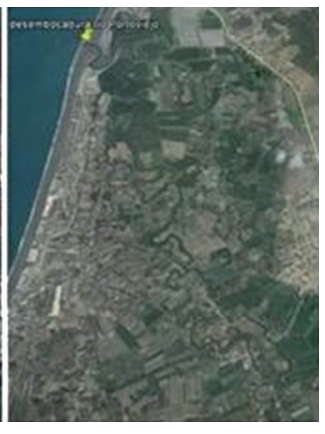

C

Fig. 5. Evolución de la cobertura de bosques ribereños en la cuenca baja del río Portoviejo, Manabí, Ecuador. En la escena (a) del año 1970 ya se aprecia una notable intervención del paisaje en ambas márgenes del río. Esta situación se ve acentuada en las escenas (b-c) correspondientes a los años 2006 y 2014 . Fuente: Imágenes tomadas de Google Earth. 
CUADRO III. CAMBIOS DE USO DEL SUELO EN LAS CUENCAS ESTUDIADAS. DATOS ESTIMADOS POR LOS AUTORES.

\begin{tabular}{|c|c|c|c|c|c|c|c|c|}
\hline \multirow[t]{2}{*}{ Tipo de Cambio } & \multicolumn{3}{|c|}{$\begin{array}{c}\text { Quebrada Chilán } \\
36104.12 \text { (ha) }\end{array}$} & \multicolumn{3}{|c|}{$\begin{array}{l}\text { Río Portoviejo } \\
213203.73 \text { (ha) }\end{array}$} & \multirow[t]{2}{*}{ Total } & \multirow[t]{2}{*}{$\%$} \\
\hline & $1990-2000$ & $2000-2008$ & Total & $1990-2000$ & $2000-2008$ & Total & & \\
\hline Bosque a área poblada & 1.44 & 5.9 & 7.34 & 1.43 & 18.54 & 19.97 & 27.31 & 0.47 \\
\hline $\begin{array}{l}\text { Bosque a área sin } \\
\text { cobertura vegetal }\end{array}$ & & & & 15.14 & 13.59 & 28.73 & 28.73 & 0.50 \\
\hline $\begin{array}{l}\text { Bosque a cuerpo de } \\
\text { agua artificial }\end{array}$ & & & & 1.53 & 110.61 & 112.14 & 112.14 & 1.94 \\
\hline Bosque a cultivo anual & & & & 101.52 & 1010.07 & 1111.59 & 1111.59 & 19.23 \\
\hline $\begin{array}{l}\text { Bosque a mosaico } \\
\text { agropecuario }\end{array}$ & 238.44 & 0.09 & 238.53 & 2303.65 & 402.3 & 2705.95 & 2944.48 & 50.93 \\
\hline Bosque a pastizal & & & & 7.74 & 1555.12 & 1562.86 & 1562.86 & 27.03 \\
\hline Totales & 239.88 & 5.99 & 245.87 & 2431.01 & 3110.23 & 5541.24 & 5787.11 & 100.00 \\
\hline
\end{tabular}

En este caso particular, la disponibilidad casi permanente de agua en el río Portoviejo y la construcción de infraestructuras para el riego han facilitado en gran medida la intervención espacial irracional, indiscriminada y descontrolada, que ha destruido la mayor parte de los bosques de la cuenca en su parte baja. La disponibilidad de infraestructura de riego para la intensificación de actividades agropecuarias con la consecuente degradación de bosques, se debe a los elevados registros de lluvia en la parte alta, así como también por los cuantiosos aportes que se producen al trasvasar agua de las presas Daule-Peripa y La Esperanza, hacia el embalse de Poza Honda. Así, la utilización de un recurso natural como el agua ha

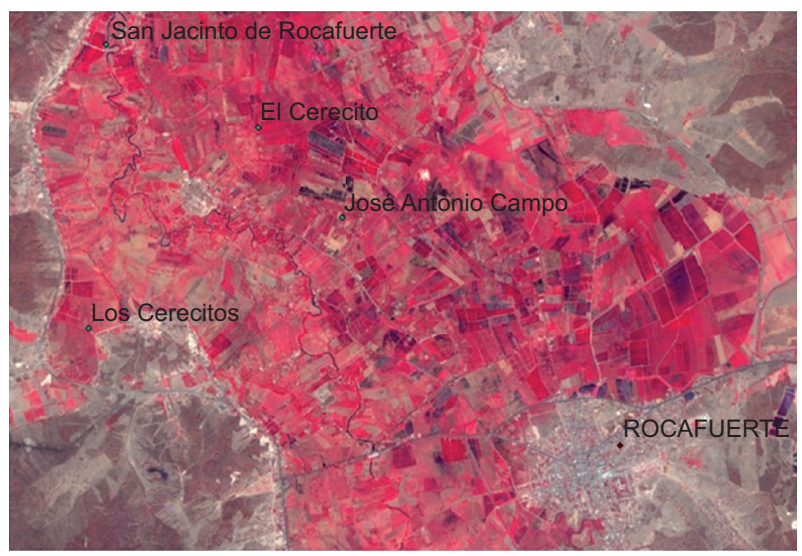

Fig. 6. Imagen RapidEye de noviembre de 2009 en falso color $(5,3,2)$. Se observa un mosaico agropecuario en las proximidades de Rocafuerte, evidenciando la presión del uso agrícola del suelo sobre las márgenes del río, donde se ha eliminado completamente el bosque ribereño.

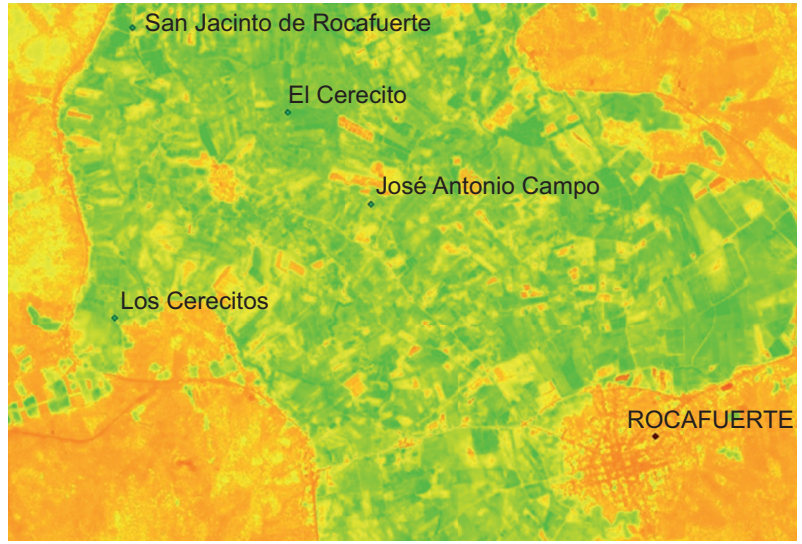

Fig. 7. Índice Normalizado de Diferencia de Vegetación, donde destaca la inexistencia de una estructura boscosa en las márgenes del río Portoviejo. Los tonos verdes representan áreas cultivadas y pastizales, y los tonos amarillo y marrón, áreas muy intervenidas y/o desprovistas de vegetación.

contribuido a la destrucción de los ecosistemas. Igualmente, la existencia de condiciones topográficas favorables (pendientes suaves) ha facilitado el desarrollo de cultivos hortícolas y de cereales en el tramo inferior del río Portoviejo, coincidiendo con lo que señalan Alatorre et al. (2014) en un estudio de la dinámica vegetal en la región central de Chihuahua, México.

Adicionalmente, en ciertos casos, los agricultores utilizan el fuego como práctica agrícola, afectando a la productividad del suelo y destruyendo igualmente parte de la vegetación ribereña. Todo ello agrava los niveles de degradación, pues la repelencia al agua (hidrofobia) inducida o intensificada por el fuego se 
considera un factor determinante en la generación de flujos de escorrentía y en los procesos erosivos repetidamente observados, por ejemplo, en las áreas quemadas del noroeste de España (Benito et al. 2014).

Durante las visitas de campo efectuadas al área de estudio del río Portoviejo se observó un patrón muy irregular en cuanto a la geometría de los pequeños fragmentos de bosque ribereño. En algunos sectores los parches de pequeños árboles tienen $10 \mathrm{~m}$ de ancho, mientras que en otros se pueden apreciar parches de $15,20,30$ y hasta $50 \mathrm{~m}$ de ancho. La variabilidad en la anchura de tales fragmentos se asocia a diferentes causas, por ejemplo, el nivel de conciencia de algunos productores en particular, que piensan que proteger las riberas del río ayuda al ambiente y a preservar el agua, contribuyendo además a resguardar sus cultivos durante eventos de inundación. En otros casos, por el contrario, los agricultores destruyen el bosque hasta la propia orilla del río, argumentando que son tierras de su propiedad, tal como señalaron Ortiz-Arrona et al. (2005) en México, aún con el conocimiento de que esas tierras son de propiedad federal.

\section{Consecuencias de la destrucción del bosque ri- bereño}

Los bosques de ribera son de importancia estratégica desde el punto vista socioeconómico, pues tradicionalmente proveen de servicios ecosistémicos a buena parte de la población residente en el área de estudio, a la que le ha suministrado frutos, maderas y materia prima para la elaboración de productos de uso cotidiano. Pero también guardan un gran interés desde un punto de vista ecológico, al actuar como hábitat de muchas especies y proteger el suelo de procesos de erosión lateral producida por la propia corriente fluvial (Herrero 2007), especialmente cuando los cursos de agua experimentan grandes crecidas y se desbordan.

Sánchez et al. (2004), señalan que diversos estudios han demostrado la importancia de esos bosques para la conservación de la biodiversidad, pues proporcionan alimento y refugio para la fauna silvestre y albergan gran cantidad y variedad de invertebrados. Agregan los mismos autores que la cobertura de árboles de estos ecosistemas también tiene un valor ecológico al proveer refugio para muchos animales que han perdido sus hábitats originales, incluyendo algunos de importancia económica, como las especies cinegéticas. Además, la cobertura de árboles funciona como un conjunto de corredores que facilitan el movimiento de animales, al tiempo que actúan como zona de amortiguamiento y de mejora de las condiciones de microclima local. Al destruir estas formaciones ve- getales, todos los beneficios descritos desaparecen de forma inmediata. Por otro lado, la eliminación de los bosques ribereños en la cuenca baja de la quebrada Chilán y el río Portoviejo ha dado lugar al aumento de la erosión del suelo y de los niveles de riesgo de inundación, materializado con la aparición más frecuente de estos eventos en los últimos años.

\section{Acciones y estrategias para recuperar el bosque de ribera y para coadyuvar a frenar los procesos que conducen a su degradación en diferentes sectores del curso bajo del río Portoviejo}

Dentro del conjunto de acciones y estrategias a emplear para recuperar los bosques de ribera en el área estudiada, sugerimos en primer lugar, la formulación y posterior ejecución de un plan de restauración ecológica, pues según Hernández et al. (2012), éste es capaz de ayudar a que un ecosistema degradado, si el nivel de alteración lo permite, recupere parte de las propiedades que presentaba antes de ocurrir su alteración.

Un proceso de restauración no es una actividad sencilla, pues no consiste únicamente en reforestar, se trata más bien de cumplir un protocolo riguroso con el fin de asegurar el éxito. En ese contexto, es necesario involucrar actividades de rehabilitación y mitigación, que consisten en la reincorporación de especies originales que favorecen las funciones clave del ecosistema. Bradshaw (1996) agrega que es pertinente el establecimiento de mejoras integrales que abarquen recuperación y rehabilitación del ecosistema degradado.

De acuerdo con los resultados de esta investigación y teniendo en cuenta las condiciones geoecológicas del área, proponemos además, para formular el plan de restauración, las siguientes actuaciones:

Diseñar y ejecutar un diagnóstico de las condiciones ecológicas del paisaje e identificar las causas que provocaron la destrucción o fragmentación del bosque ribereño; reconocer potenciales disturbios y posibles perturbaciones ecológicas a fin de erradicarlas o minimizarlas. Si esto no se hace, no es posible que las acciones que se implementen para recuperar los bosques sean exitosas. En todo caso, quienes vayan a diseñar un plan de restauración ecológica deben considerar que es pertinente:

a. Delimitar con la ayuda de tecnologías de SIG y sensores remotos, las áreas prioritarias para la restauración en atención a diferentes variables, como por ejemplo, grado de destrucción, posibilidades de restauración, acceso, costos económicos y otros. 
b. Preparar de forma previa el terreno con aplicaciones de abono en los sitios donde se van a introducir las plántulas a fin de asegurarles la disponibilidad de nutrientes y su posterior desarrollo. Recomendamos que las plantas seleccionadas para la siembra sean preferentemente de las mismas especies que existían en el bosque anterior a su degradación. Entre otras la ceiba y el algarrobo, puesto que ayudan a recuperar más rápidamente las funciones ecosistémicas al aportar hojarasca y facilitar los procesos de fijación del suelo por la profundidad de sus raíces. Además contribuyen a la regulación del microclima y en el caso particular del algarrobo, favorece el retorno de la fauna pues es una leguminosa de la que se alimentan distintas especies, las cuales consecuentemente contribuirán en la propagación de las semillas, mecanismo que según Meli et al. (2015), ayuda a que el proceso de restauración ecológica sea exitoso. De igual forma, estas plántulas o semillas pueden obtenerse de remanentes de bosques cercanos similares a los destruidos, de los cuales, en la parte baja de la cuenca, según se aprecia en las imágenes de satélite, todavía quedan algunos.

c. Asimismo, resulta importante evaluar los costos de producción y trasplante de distintos tipos de plántulas, descartando la posibilidad de que sean sólo especies nativas las que han de introducirse, pues según Meli et al. (2014), en determinados casos esto puede ser económicamente más costoso $\mathrm{y}$, por tanto, convertirse en una limitación para las comunidades locales que quieran adelantar los procesos de restauración. Por esta razón es conveniente evaluar la posibilidad de incluir otros tipos de especies.

d. Es importante también tomar en cuenta otros costos para asegurar la viabilidad y el éxito de la restauración. Por ejemplo, Fuentealba y MartínezRamos (2014), plantean conveniente estimar el valor de la mano de obra, como también los materiales utilizados para el deshierbe y para proporcionar protección a las plántulas trasplantadas con cercas de alambre, las cuales además, requieren inversiones de mantenimiento.

e. Partiendo del hecho que las especies vegetales seleccionadas tienen propiedades útiles para la gente, se recomienda la participación activa de las comunidades con el objeto de facilitar el proceso de restauración de los ecosistemas degradados, tal como lo sugieren Meli et al. (2014).

f. Tener especial cuidado en la selección del momento de siembra de los árboles, siendo más recomendable hacerlo al inicio de la temporada de lluvias. Eso disminuye los gastos que pudiera ocasionar el riego de las plántulas sembradas y ayudaría a asegurar el crecimiento de las mismas. Para el área de estudio, la siembra deberá realizarse desde finales del mes de diciembre hasta mediados del mes de enero.

g. Construir corredores de vegetación, especialmente en el tramo final del curso del río Portoviejo, a corta distancia de la desembocadura en el Pacífico, pues allí existen algunos parches de vegetación boscosa. La construcción de estos corredores de vegetación tendría como función crear conectividad entre los diversos fragmentos de bosque, de manera que las especies animales y vegetales puedan tener un hábitat más amplio y con ello aumentar las posibilidades de dispersión de semillas y propagación de plantas. Esta actividad se puede desarrollar con relativa facilidad en el caso que nos ocupa, pues las distancias entre un fragmento y otro son, en general, pequeñas.

h. Construir trampas de sedimento, disipadores de energía y fajas forestales hidrorreguladoras en los sitios más críticos del área de estudio, es decir, aquellos que cuentan con una conocida historia de eventos de inundación. Con ello se daría protección ante potenciales inundaciones a los núcleos habitados que presentan vulnerabilidad, entre ellos La Boca, San Silvestre, Corre Agua y Puerto Higuerón, ubicados en la cuenca baja del río Portoviejo.

i. Construir escombreras para dar protección y refugio a la fauna mediana y pequeña. Éstas deben construirse lo más alejadas posible de los centros poblados, teniendo en cuenta que los animales puedan tener fácil acceso a las mismas.

j. Establecer como actividad permanente, acciones de monitoreo de la evolución del plan en todas sus fases. Con ello se podrán retomar acciones y establecer correcciones sobre la marcha.

k. Finalmente, un conjunto de señales, dentro y fuera de las áreas sometidas a restauración, dará mayor proyección al plan de actuación. En todo caso, el conjunto de acciones de restauración debe contar con el apoyo y la participación activa de los actores sociales involucrados en los cambios del paisaje, fundamentalmente los propietarios y productores agrícolas de las localidades de San Jacinto, San Clemente, El Pueblito, Charapotó, Cañitas, San Eloy, El Higuerón de Rocafuerte y Crucita, entre otros. A ellos se debe sensibilizar con la implementación de talleres acerca de la importancia que representa la preservación y restauración de los bosques ribereños, evitando el error de no tener en cuenta la opinión de estos 
actores, que deben formar parte del equipo que lleve a cabo las labores de restauración.

\section{CONCLUSIONES}

Los bosques ribereños del río Portoviejo y la quebrada Chilán proveen de servicios ecosistémicos a buena parte de la población asentada en localidades de Jaramijó, Crucita, El Higuerón de Rocafuerte y Charapotó, entre otros. En este caso, estos bosques revisten un gran interés ecológico y social, porque también actúan como hábitat de muchas especies, proporcionan madera y leña como combustible y protegen el suelo de procesos de erosión lateral producida por la propia corriente fluvial.

Los resultados del trabajo aportan información sobre el comportamiento hidrológico del río Portoviejo y la quebrada Chilán. Por ejemplo, se ilustra el hecho que estos cursos de agua han experimentado de forma recurrente importantes crecidas, con desbordamiento e inundaciones, las cuales han tenido consecuencias fatales para la población, pero además fuertes impactos sobre el bosque ribereño. En consecuencia, esta información resulta de utilidad para los pobladores de localidades afectadas, pero además para entes gubernamentales, especialmente al momento de diseñar estrategias con miras a disminuir los efectos resultantes de aquellos fenómenos, como también cuando sea posible diseñar un plan de restauración ecológica en sectores donde los ecosistemas de bosque ribereño han sido más degradados.

Como ya se mencionó, la información obtenida señala que cíclicamente se producen desbordamientos e inundaciones. Por lo tanto, en el marco de proyectos de restauración, sería ideal la reforestación con especies autóctonas, cuyas propiedades funcionales permitan el desarrollo y fijación del suelo, como también un aumento rápido de biomasa a orillas del río, a fin de minimizar la fuerza de la corriente de agua y evitar males mayores al producirse las inundaciones.

En el caso de la quebrada Chilán las perturbaciones en el bosque ribereño se aprecian de manera temporal por causas naturales, relacionadas con crecidas torrenciales que son una consecuencia de la manifestación del fenómeno El Niño.

La llanura de inundación y el propio cauce del río Portoviejo, en la cuenca baja, han sido utilizadas para uso residencial y agropecuario de forma permanente, extensiva y con poca planificación, lo cual ocasiona la desaparición casi total del bosque de ribera.

Por lo anterior, se concluye también, que es de suma importancia emprender acciones para frenar los procesos de degradación del bosque de ribera, pero también para su recuperación. En este caso,luce necesario, como ya se planteó, materializar las acciones y sugerencias especificadas para la restauración indicadas en la sección de resultados y discusión.

\section{REFERENCIAS}

Alatorre L., Miramontes-Beltrán S., García-Peña A., Díaz-Caravantes R. y Bravo L. (2014). Evolución de la dinámica vegetal mediante una serie de imágenes Landsat TM (1986-2011): Región central de Chihuahua, México. Cuad. Invest. Geogr. 40, 449-476.

Ambrosio G., González J. y Arévalo V. (2007). Corrección radiométrica y geométrica de imágenes para la detección de cambios en una serie temporal. Departamento de Ingeniería de Sistemas y Automática. Universidad de Málaga, Málaga, España, 346 pp.

Baihua F. y Burgher I. (2015). Riparian vegetation NDVI dynamics and its relationship with climate, surface water and groundwater. Journal of Arid Environments 113, 59-68. DOI: 10.1016/j.jaridenv.2014.09.010

Benito E., Varela M. y Rodríguez-Alleres M. (2014). Efectos de los incendios forestales en la erosionabilidad de los suelos en Galicia. Cuad. Invest. Geogr. 40, 353-370.

Bolívar S. (2012). Caracterización geomorfológica y sedimentológica de la bahía de Jaramijó, en la provincia de Manabí. Acta Oceanográfica del Pacífico 17, 205-220.

Bradshaw A. (1996). Underlying principles of restoration. Can. J. Fish. Aquat. Sci. 53, 3-9.

Brizuela A., Aguirre C. y Velazco I. (2007). Aplicación de métodos de corrección atmosférica de datos Landsat 5 para análisis multitemporal. Memorias. Teledetección. Hacia un mejor entendimiento de la dinámica global y regional. Madrid, España. 21 al 26 de septiembre, 2007. CD-ROM.

Cedeño R. (2010). Identidad manteña. Universidad Laica Eloy Alfaro de Manabí. Manta, Ecuador, 225 pp.

Chuvieco E. (2000). Fundamentos de teledetección espacial. Ediciones Rialp, Madrid, España, 568 pp.

Compton T., Denelle G. y Jon D. (2004). NASA's global orthorectified landsat data set. Photogramm. Eng. Remote Sens. 70, 313-322. DOI: 10.14358/PERS.70.3.313

Fuentealba B. y Martínez-Ramos M. (2014). Transplanting native tree seedlings to enrich tropical live fences: an ecological and socio-economic analysis. Agroforest Syst. 88, 221-236. DOI: 10.1007/s10457-013-9669-y

Gobierno Autónomo Descentralizado Municipal del Cantón Montecristi (2011). Plan de Desarrollo 
Cantonal y Plan de Ordenamiento Territorial del Cantón Montecristi 2011-2016. Gobierno Autónomo Descentralizado Montecristi, Montecristi, Ecuador, 209 pp.

Gond V., Bartholome E., Ouattara F., Nonguierma A. y Bado L. (2004). Surveillance et cartographie des plan d'eau et des zones humides et inondables en régions arides avec l'instrument VEGETATION embarqué sur SPOT 4. Int. J. Remote Sens. 25, 987-1004.

DOI: 10.1080/0143116031000139908

Hernández A., Langdon B. y Ramírez P. (2012). Plan de restauración del bosque nativo Arauco. Segunda versión, Gerencia de desarrollo forestal Arauco, Santiago de Chile, Chile. 187 pp.

Herrero J. (2007). Fajas Forestales Hidrorreguladoras. Situación e importancia. Rev. Agricultura Orgánica $13,40-42$

Hum Y., Lai K., Mohamad S. y Maheza I. (2014). Multiobjectives bihistogram equalization for image contrast enhancement. Complexity 20, 22-36.

DOI: $10.1002 /$ cplx.21499

INEC (2010). Resultados del censo de población y vivienda Censo 2010, Fascículo Provincial. Instituto Nacional de Estadística y Censos. Manabí, Ecuador [en línea]. http://es.scribd.com/doc/100658998/Manabiresumen-Censo-Poblacion-y-Vivienda- 2010\#scribd $12 / 05 / 2015$

INAMHI (2014). Anuarios Meteorológicos 1991-2012. Instituto Nacional de Meteorología. Quito, Ecuador, $149 \mathrm{pp}$.

Jacques D., Kergoat L., Hiernaux P., Mougin E. y Defourny P. (2014). Monitoring dry vegetation in semi-arid areas with MODIS SWIR bands. Remote Sens. Environ. 153, 40-49. DOI: 10.1016/j.rse.2014.07.027

MAE (2010). Mapa histórico de la deforestación del Ecuador continental. Ministerio del Ambiente del Ecuador. Quito, Ecuador, 46 pp.

Meli P., Hernández G., Castro E. y Carabias J. (2015). Vinculando paisaje y parcela: un enfoque multiescala para la restauración ecológica en áreas rurales. Investigación Ambiental Ciencia y Política Pública 7, 44-53.

Meli P., Martínez-Ramos M., Rey-Benayas J. y Carabias J. (2014). Combining ecological, social, and technical criteria to select species for forest restoration. Appl. Veg. Sci.17, 744-753. DOI: 10.1111/avsc. 12096

Milton J. (2012). Manta, su historia y progreso. Editorial Libromanta. Manta, Ecuador, 298 pp.

Organización Desinvetar (2013). Sistema de información de desastres y emergencias de Ecuador [en línea]. https://online.desinventar.org/ 12/05/2015

Ortiz-Arrona C., Gerritsen, P., Martínez Rivera M., Allen A. y Snoep M. (2005). Restauración de bosques ribereños en paisajes antropogénicos en el occidente de México [en línea]. http://www.globalrestorationnetwork.org/uploads/files/CaseStudyAttachments/132 estudio-de-caso.pdf 25/06/2015

Pourrut P. (1983). Los Climas del Ecuador. Fundamentos explicativos. Estudio realizado en el marco de un convenio entre la ORSTOM y PRONAREG. Centro Ecuatoriano de Investigación Geográfica, Quito, Ecuador, $87 \mathrm{pp}$.

Pourrut P. (1995). El agua en el Ecuador. Clima, Precipitaciones, Escorrentía. Corporación Editora Nacional. Quito, Ecuador, 378 pp.

República del Ecuador (2008). Constitución de la República del Ecuador [en línea]. http://www.oas.org/juridico/ PDFs/mesicic4_ecu_const.pdf 12/05/2015

Ruíz D. (2004). La biodiversidad en la ecorregión de los Llanos de Venezuela y las prioridades para su conservación. Ecosistemas 13, 124-129.

Sánchez D., López M., Medina A., Gómez R., Harvey C., Vílchez S., Hernández B., López F., Joya M., Sinclair F. y Kunth S. (2004). Importancia ecológica y socioeconómica de la cobertura arbórea de un paisaje fragmentado de bosque seco de Belén Rivas, Nicaragua. Encuentro 68, 7-23.

SENPLADES (2010a). Lineamientos para la planificación del desarrollo y el ordenamiento territorial. Estrategias para el fortalecimiento del Sistema Nacional Descentralizado de Planificación Participativa. Secretaría Nacional de Planificación y Desarrollo, Quito, Ecuador, 40 pp.

SENPLADES (2010b). Agenda zonal para el buen vivir. Propuestas de desarrollo y lineamientos para el ordenamiento territorial. Secretaría Nacional de Planificación y Desarrollo. Quito, Ecuador, 78 pp.

SENPLADES (2013). Plan Nacional de Desarrollo / Plan Nacional para el Buen Vivir 2013-2017. Secretaría Nacional de Planificación y Desarrollo. Quito, Ecuador, $602 \mathrm{pp}$.

Song C., Woodcock K., Seto M. y Lenney S. (2001). Classification and Change Detection Using Landsat TM Data: When and How to Correct Atmospheric Effects? Remote Sens. Environ. 75, 230-244.

DOI: 10.1016/S0034-4257(00)00169-3

Sun W., Shao Q., Liu J. y Zhai J. (2014). Assessing the efects of land use and topography on soil erosion Loess Plateau in China. Catena 121, 151-163.

DOI: 10.1016/j.catena.2014.05.009

Thielen D., Cevallos J., Erazo T., Zurita I., Figueroa J., Quintero J., Matute N., Velásquez G. y Puche M. (2015). Dinámica de eventos climáticos extremos en la cuenca del río Portoviejo, Manabí. La Técnica 14, 80-91.

Treviño E., Cavazos C. y Aguirre O. (2001). Distribución y estructura de los bosques de galería en dos ríos del centro sur de Nuevo León. Madera y Bosques 7, 13-25. 
Universidad Nacional de San Luis (2011). Elementos de geología -principios de geociencias -geología. Apuntes para trabajos prácticos [en línea]. http:// www.unsl.edu.ar/ geo/materias/Elementos_de_Geologia/documentos/contenidos/apoyo_teorico/APU2011-Fotointerpret.pdf 14/05/2015
Zevallos O. (2004). Proyecto gestión del riesgo ENSO en América latina. Investigación comparativa, información y capacitación desde una perspectiva social. Informe técnico. Patrones y procesos de configuración LA RED [en línea]. http://www.cambioglobal.org/enso/informes/ anho3/SIA-enso-2001_2002.pdf 26/06/2015. 